\title{
Stability of the Global Ocean Circulation: Basic Bifurcation Diagrams
}

\author{
Henk A. Dijkstra \\ Institute for Marine and Atmospheric Research Utrecht, Utrecht University, Utrecht, Netherlands, and Department of Atmospheric \\ Science, Colorado State University, Fort Collins, Colorado \\ WiLBERT WeIJER \\ Scripps Institution of Oceanography, University of California, San Diego, La Jolla, California
}

(Manuscript received 4 April 2003, in final form 20 October 2004)

\begin{abstract}
A study of the stability of the global ocean circulation is performed within a coarse-resolution general circulation model. Using techniques of numerical bifurcation theory, steady states of the global ocean circulation are explicitly calculated as parameters are varied. Under a freshwater flux forcing that is diagnosed from a reference circulation with Levitus surface salinity fields, the global ocean circulation has no multiple equilibria. It is shown how this unique-state regime transforms into a regime with multiple equilibria as the pattern of the freshwater flux is changed in the northern North Atlantic Ocean. In the multiple-equilibria regime, there are two branches of stable steady solutions: one with a strong northern overturning in the Atlantic and one with hardly any northern overturning. Along the unstable branch that connects both stable solution branches (here for the first time computed for a global ocean model), the strength of the southern sinking in the South Atlantic changes substantially. The existence of the multipleequilibria regime critically depends on the spatial pattern of the freshwater flux field and explains the hysteresis behavior as found in many previous modeling studies.
\end{abstract}

\section{Introduction}

The response of the global ocean circulation to changes in the surface buoyancy and wind forcing is one of the important problems in climate research. The momentum input through the wind stress is the main driving mechanism of the flow in the upper layers of the ocean, in particular for western boundary currents such as the Gulf Stream, Kuroshio, and the Agulhas Current. The buoyancy input through the surface heat and freshwater flux is crucial for the existence and structure of the stratification in the ocean and, hence, for the mainly density-driven deep flow (Schmitz 1995).

A useful framework to understand the global circulation is the "Ocean Conveyor" (Gordon 1986). In this view, the deep and surface flows in the Atlantic, Pacific, and Indian Ocean basins are connected through currents in the Southern Ocean. The North Atlantic is one of the central regions in this framework; here the northward flowing surface water becomes dense enough to induce formation of North Atlantic Deep Water

Corresponding author address: Henk A. Dijkstra, Department of Atmospheric Science, Colorado State University, Fort Collins, CO 80526.

E-mail: dijkstra@atmos.colostate.edu
(NADW). This water mass moves southward and can be traced at depth far south in the Atlantic basin. Because of the existence of this overturning branch of the Atlantic circulation, about $1.5 \mathrm{PW}$ of heat is transported northward at $24^{\circ} \mathrm{N}$ (Hall and Bryden 1982; Ganachaud and Wunsch 2000).

More and more evidence emerges that is in favor of an active role of the ocean circulation in past climate changes (Clark et al. 2002). The North Atlantic circulation has a special role because it appears sensitive to the amplitude and pattern of the surface buoyancy forcing. There is much evidence that the freshwater input at particular locations has caused, at several instances in the geological past, a temporary slowdown of the North Atlantic circulation (Broecker 2000). A famous example is the onset of the Younger Dryas cooling event, about 12000 years ago, which (likely) started with a catastrophic input of freshwater through diversion of Lake Agassiz from the Mississippi basin to the St. Lawrence drainage (Rooth 1982).

In addition, the response of the ocean circulation to changes in the surface forcing is a central factor in the different climate change scenarios computed with current climate models that investigate the impacts of increased atmospheric greenhouse gas concentrations (Houghton et al. 2001). The increased radiative forcing 
changes the hydrological cycle to induce more precipitation in the North Atlantic, which may slow down the meridional overturning circulation. Apart from changes in the freshwater flux, also changes in the oceanatmosphere heat flux may affect the meridional overturning. The degree of the slowdown depends on the model configuration and on properties of the mean climate state (Manabe and Stouffer 1999; Clark et al. 2002).

Stability properties of the global ocean circulation have first been addressed with ocean-only models. The pioneering results of Bryan (1986) revived the interest in multiple flow patterns of the Atlantic Ocean circulation already suggested by Stommel (1961). One of the issues that has been extensively investigated is the stability of the global ocean flow that arises under prescribed (observed) sea surface salinity (SSS) and sea surface temperature (SST) fields, with restoring time scales $\tau_{S}$ and $\tau_{T}$, respectively. Tziperman et al. (1994) study the stability of these flows under mixed boundary conditions (with a diagnosed freshwater flux) and found that the circulation is unstable if $\tau_{S}$ is small enough. In the latter case, the freshwater flux (evaporation-precipitation) becomes strongly negative in the northern North Atlantic, which inhibits northern sinking.

The sensitivity of the global ocean circulation to buoyancy input into the North Atlantic has also been investigated by adding freshwater at a very slow rate such that quasi-steady states are monitored in time (Rahmstorf 1995; Prange et al. 2002). In this way, a first impression of the stable equilibria of the global ocean circulation can be obtained (Rahmstorf 2000). In a global ocean general circulation model (OGCM) coupled to a zeroth-order atmosphere model, it is found that when this freshwater input is strong enough, the Atlantic meridional overturning circulation (MOC) strongly decreases. When the freshwater forcing is reversed from this weak MOC state, hysteresis behavior occurs because this state can be maintained under substantial negative freshwater flux (i.e., input of salt) anomalies. This has lead to the suggestion that the underlying bifurcation diagram is qualitatively similar to that in the simple two-box model suggested in Stommel (1961).

Although there are models in which no weak MOC state is found in the parameter regime investigated (Schiller et al. 1997), the multiple-equilibria structure appears to be a robust feature in coupled oceanatmosphere models. Manabe and Stouffer (1999), for example, showed that a weak MOC state is reached as a result of a certain freshwater perturbation in case the ocean model has a small vertical mixing coefficient of heat and salt that varies with depth. Note, however, that with a larger constant vertical mixing coefficient the MOC reduced substantially but recovered after some time. Tziperman (1997) demonstrates that the occurrence of a weak MOC state depends strongly on the mean salinity field in the North Atlantic Ocean. In these model studies, the weak MOC state has deepwater formation in the Southern Ocean and upwelling in the northern North Atlantic.

The multiple-equilibria regime may be a very important characteristic of the global ocean circulation and likely of the climate system. Its sensitivity to representations of physical processes in ocean models is crucial to determine whether relatively rapid climate changes, as recorded in ice-core records (Alley et al. 1997), can be attributed to changes in the global ocean circulation. It is also important for assessing the probability that a future weakening of the MOC will occur as the concentration of greenhouse gases is increasing. Extensive sensitivity studies have only been performed in intermediate complexity models, such as zonally averaged models (Schmittner and Stocker 1999). Recently, Prahl et al. (2003) performed a sensitivity study of the hysteresis behavior with respect to the vertical eddy diffusivity $\left(K_{V}\right)$ in a global ocean model. They show that the hysteresis behavior exists over a large range of values of $K_{V}$ and that the width of the hysteresis loop becomes smaller with decreasing $K_{V}$.

In this paper, the first explicit computation of the bifurcation diagram explaining the hysteresis behavior of the global ocean circulation and its sensitivity to $K_{V}$ is presented. The techniques of numerical bifurcation theory are now in a stage that one is able to perform these computations for a low-resolution OGCM $\left(4^{\circ}\right.$ horizontal resolution and 12 vertical layers) coupled to an energy-balance atmospheric model. For a similar configuration as used in Rahmstorf (1995), the complete bifurcation structure is computed including the branch of unstable solutions, which is necessarily present in the bifurcation diagram. The connection to earlier bifurcation diagrams in more idealized configurations, that is, an Atlantic-only configuration, is presented elsewhere (Dijkstra and Weijer 2003).

\section{Model and methods}

The behavior of the global ocean model described in section $2 \mathrm{a}$ is analyzed in a manner complementary to traditional model studies (Rahmstorf 1995; Tziperman, 2000). The complementarity of the approach is described in section $2 \mathrm{~b}$ using a simple example. In section $2 \mathrm{c}$, the numerical techniques are outlined and, as explained in section $2 \mathrm{~d}$, they pose some restrictions on the parameter regime in the ocean model that can be handled.

\section{a. Model}

The description of the fully implicit global ocean model used in this study is presented in Weijer et al. (2003) to which the reader is referred for full details. The governing equations of the ocean model are the hydrostatic, primitive equations in spherical coordinates on a domain that includes full continental geom- 
etry as well as bottom topography. The ocean velocities in eastward and northward directions are indicated by $u$ and $v$, the vertical velocity is indicated by $w$, the pressure by $p$, and the temperature and salinity by $T$ and $S$.

The horizontal resolution of the model is about $4^{\circ}$ [a $96 \times 38$ grid in a domain $\left(85.5^{\circ} \mathrm{S}-85.5^{\circ} \mathrm{N}, 0^{\circ}-360^{\circ}\right.$ longitudinally)], and the grid has 12 levels in the vertical direction. The vertical grid is nonequidistant with the most upper (lowest) layer having a thickness of $50 \mathrm{~m}$ $(1000 \mathrm{~m})$, respectively. In the present version of the ocean model, we have followed the configuration of the Geophysical Fluid Dynamics Laboratory (GFDL) Modular Ocean Model (MOM), as described in England (1993) and used in Rahmstorf (1996), very closely. Vertical and horizontal mixing of momentum and heat/salt are represented by a Laplacian formulation with prescribed eddy viscosities $A_{H}$ and $A_{V}$ and eddy diffusivities $K_{H}$ and $K_{V}$, respectively.

The oceanic flow is forced by the annual-mean wind stress as given in Trenberth et al. (1989). The upper ocean is coupled to a simple energy-balance atmospheric model as in Dijkstra et al. (2003), in which only the heat transport is modeled (no moisture transport); a short summary of this model is provided in the appendix. The freshwater flux will be prescribed in each of the results below. As in low-resolution OGCMs, the surface forcing is represented as a body forcing over the upper layer. On the continental boundaries, no-slip conditions are prescribed, and the heat and salt fluxes are zero. At the bottom of the ocean, both the heat and salt fluxes vanish and slip conditions are assumed.

\section{b. Continuation methods}

Since many readers may (still) be unfamiliar with continuation methods, a brief introduction into this approach in relation to more traditional ocean modeling is given here.

Suppose one is interested in the changes of equilibrium flows of a model (here the low-resolution global ocean model) with respect to changes in the forcing conditions, say the surface freshwater flux

$$
F_{S}=F_{S}^{1}+\lambda F_{S}^{2}
$$

where $\lambda \in[0,1]$ is a parameter controlling the freshwater flux (its pattern and amplitude) and the $F_{S}^{i}(i=$ $1,2)$ are prescribed patterns.

In traditional ocean models, this problem would be approached as a set of initial value problems for several chosen values of $\lambda$, for example, $\lambda=0.25,0.5,0.75$, and $\lambda=1.0$. For the first value of $\lambda$, a motionless flow with interpolated Levitus values for temperature and salinity can be taken as an initial condition at time $t=t_{0}$. To determine the equilibrium state, an initial value problem is solved in which the solution at time $t=t_{n+1}$ is computed from the solution at time $t=t_{n}$.

To solve this initial value problem explicit techniques are commonly used, such as leapfrog or Adams-
Bashforth. Mode splitting, where the barotropic mode is treated differently than the baroclinic modes, is often used, and sometimes the time steps for the tracers and momentum are taken differently. In explicit methods, the time step is limited by numerical stability according to well-known criteria. After many time steps (a typical time step is $1 \mathrm{~h}$ and one has to integrate several thousands of years), one ends up with an equilibrium solution for which all fields do not change in time anymore. Next, this procedure is repeated for the other values of $\lambda$. One could either choose the same initial conditions as for the first value of $\lambda$ or one can make use of equilibrium solutions computed at earlier values of $\lambda$.

As an example, consider the two-dimensional system of differential equations given by

$$
\begin{aligned}
& \frac{d x}{d t}=\lambda-x^{2} \quad \text { and } \\
& \frac{d y}{d t}=x-y .
\end{aligned}
$$

For each $\lambda>0$, when solved as an initial value problem, $x(t)$ and $y(t)$ are followed in time until a steady state is reached. If for these values of $\lambda, x(t)$ is plotted versus time, a plot as in Fig. 1a results. However, for the question at hand, that is, the sensitivity of the equilibria versus $\lambda$, only the end points of these calculations, say $(\bar{x}, \bar{y})=\lim _{t \rightarrow \infty}[x(t), y(t)]$, are of interest and typically one would show a plot as in Fig. 1b.

The continuation methods used here are designed to directly compute the curve in Fig. 1b without going through the transient calculations as in Fig. 1a. Instead of solving the time-dependent equations, these techniques directly tackle the steady equations, that is, the model equations with the time-derivatives put to zero. For the example,

$$
\begin{aligned}
\lambda-\bar{x}^{2} & =0 \quad \text { and } \\
\bar{x}-\bar{y} & =0 .
\end{aligned}
$$

Solving directly for the latter has an important additional advantage. For the steady states, we find $\bar{x}=\bar{y}=$ $\sqrt{\lambda}$ and $\bar{x}=\bar{y}=-\sqrt{\lambda}$ for each value of $\lambda>0$. Looking at the evolution of small perturbations on these states, say

$$
x=\bar{x}+\tilde{x} \quad \text { and } \quad y=\bar{y}+\tilde{y},
$$

the linearized equations from (2) are

$$
\begin{aligned}
& \frac{d \tilde{x}}{d t}=(-2 \bar{x}) \tilde{x} \quad \text { and } \\
& \frac{d \tilde{y}}{d t}=\tilde{x}-\tilde{y} .
\end{aligned}
$$

For the solution $\bar{x}=\bar{y}=\sqrt{\bar{\lambda}}$, the perturbations will decay and hence the steady state is stable and will be found by transient integration (as in Fig. 1a). The so- 
(a)
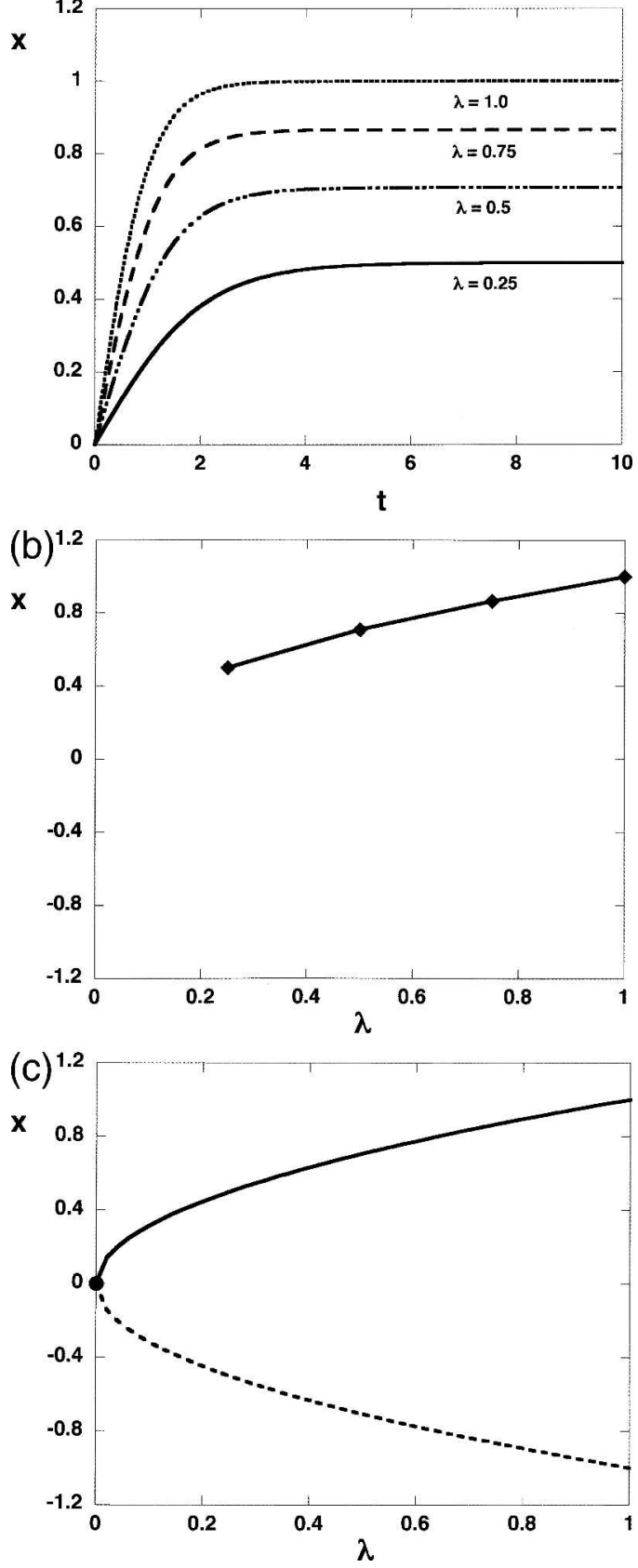

FIG. 1. Solution of the system (2) with (a) transient solutions for several values of $\lambda,(\mathrm{b})$ the steady-state values of $x$ vs $\lambda$, and (c) the complete bifurcation diagram of (2).

lution $\bar{x}=\bar{y}=-\sqrt{\lambda}$ is unstable since perturbations on it will grow and hence it will never be reached by the transient approach. However, by solving directly for the steady equations both solutions will be found-also the unstable one! The steady-state branches, as they are computed by the continuation method (described in the next section), are plotted in Fig. 1c. The so-called saddle-node bifurcation at $\bar{x}=\bar{y}=\lambda=0$ can be explicitly computed with these techniques.

\section{c. Application to the ocean model}

Going back to the ocean model, the set of partial differential equations with the appropriate boundary conditions is discretized on an $N \times M \times(L+1)$ grid. After discretization, a system of nonlinear differential equations with algebraic constraints results, which can be written as

$$
\mathbf{M} \frac{d \mathbf{u}}{d t}=\mathbf{F}(\mathbf{u}, \mathbf{q}) .
$$

Here $\mathbf{u}$ is the $d$-dimensional state vector $[d=6 \times N \times$ $M \times(L+1)]$, consisting of the unknowns $(u, v, w, p, T$, $S$ ) at each grid point, $\mathbf{q}$ is the $q$-dimensional vector of parameters, $\mathbf{F}$ is a nonlinear mapping from $R^{d} \times R^{q} \rightarrow$ $R^{d}$, and $\mathbf{M}$ is a linear operator. Since $N=96, M=38$, and $L=12$, the total dimension of the dynamical system, $d=284544$. Stationary solutions satisfy the equation

$$
\mathbf{F}(\mathbf{u}, \mathbf{q})=\mathbf{0},
$$

which is a system of $d$ nonlinear algebraic equations. Note that, for the example, in the previous section, the Eqs. (2) and (3) can also be written as (6) and (7), respectively, with $d=2$ and $q=1$.

To compute a branch of stationary solutions in a control parameter, say $\lambda$, a pseudo-arc-length method is used (Keller 1977). The branches of stationary solutions $[\mathbf{u}(s), \lambda(s)]$ are parameterized by an arc length parameter $s$. Since this introduces an extra unknown, an additional equation is needed and the tangent is normalized along the branch, for example, represented by

$$
\dot{\mathbf{u}}_{0}^{\mathrm{T}}\left(\mathbf{u}-\mathbf{u}_{0}\right)+\dot{\lambda}_{0}^{\mathrm{T}}\left(\lambda-\lambda_{0}\right)-\Delta s=0,
$$

where $\Delta s$ is the step length, the superscript $\mathrm{T}$ denotes the transpose, a dot indicates differentiation to $s$ and $\mathbf{u}_{0}$ indicates a previous solution computed for $\lambda=\lambda_{0}$.

The Newton-Raphson method is used to converge to the branch of stationary solutions. During one Newton iteration, with iteration index $k$, linear systems of the form

$$
\mathcal{I}\left(\mathbf{u}^{k}\right)\left(\begin{array}{c}
\Delta \mathbf{u}^{k+1} \\
\Delta \lambda^{k+1}
\end{array}\right)=\left(\begin{array}{c}
\mathbf{r}^{k} \\
r_{d+1}^{k}
\end{array}\right)
$$

have to be solved, where $\Delta \mathbf{u}^{k+1}$ and $\Delta \lambda^{k+1}$ are updates during the Newton-Raphson process. The quantities $\mathbf{r}^{k}$ and $r_{d+1}^{k}$ derive from (7) and (8) and are given by

$$
\begin{aligned}
\mathbf{r}^{k} & =-\mathbf{F}\left(\mathbf{u}^{k}, \lambda^{k}\right) \quad \text { and } \\
r_{d+1}^{k} & =\Delta s-\dot{\mathbf{u}}_{0}^{\mathrm{T}}\left(\mathbf{u}^{k}-\mathbf{u}_{0}\right)+\dot{\lambda}_{0}\left(\lambda^{k}-\lambda_{0}\right) .
\end{aligned}
$$

This method finds isolated steady solutions, regardless of their stability. The linear system in (9) is solved with the generalized minimal residual (GMRES) method 
(an iterative linear systems solver) using matrix renumbered incomplete LU (MRILU) as a (multigridoriented) preconditioning technique. For details on the latter methods, the reader is referred to Botta and Wubs (1999) and Dijkstra et al. (2001).

The extended system of equations in (7)-(8) for the unknowns $\mathbf{u}(s)$ and $\lambda(s)$ is needed to be able to follow solutions along saddle-node bifurcations. Remember that in the example (2), a saddle-node bifurcation occurred at $\bar{x}=\bar{y}=\lambda=0$ where the Jacobian matrix from (5), that is,

$$
\mathcal{J}=\left(\begin{array}{cc}
-2 \bar{x} & 0 \\
1 & -1
\end{array}\right)
$$

becomes singular. This singularity would make it impossible to use the Newton-Raphson method for the system (7). The extended system, with Jacobian $\mathcal{I}$ as in (9), is nonsingular, allowing one to trace branches around saddle-node bifurcations.

\section{d. Shortcomings of the model}

The GMRES and MRILU are sophisticated numerical techniques enabling one to solve for a 284544 system of nonlinear equations as arises from the discretization of the ocean model [instead of a two-equation system as in (2)]. At the moment, there are two restrictions under which this can be efficiently done for the global model: (i) a relatively large value of lateral eddy viscosity $A_{H}$ and (ii) a restriction on the use of convective adjustment.

Many explicit ocean models of $4^{\circ}$ resolution use typical values of $A_{H}=2.5 \times 10^{5} \mathrm{~m}^{2} \mathrm{~s}^{-1}$. If one considers the momentum equations in a single-hemispheric basin (Dijkstra et al. 2001), this value is far too small to resolve the Ekman boundary layer near the eastern wall. This boundary layer has a thickness $\left(A_{H} / f_{0}\right)^{1 / 2}$, where $f_{0}$ $=2 \Omega \sin \phi_{0}$ is the value of the Coriolis parameter at latitude $\phi_{0}$. General numerical practice is to resolve these boundary layers since, otherwise, wiggles are encountered.

When the implicit model is used to compute steady states with this value of $A_{H}$, wiggles are seen in the whole solution. The discretization of the Coriolis terms provides difficulties on the $\mathrm{C}$ grid because of the presence of so-called velocity modes. The problem is exacerbated in the implicit approach because the whole system of equations is solved simultaneously and there is no decoupling of barotropic and baroclinic modes. In the results below, we therefore restrict use to the C-grid case in which the value of $A_{H}=1.6 \times 10^{7} \mathrm{~m}^{2} \mathrm{~s}^{-1}$ is such that the lateral Ekman boundary layers are resolved and lateral friction damps the velocity modes.

Convective adjustment is widely used in ocean models and is easily implemented in explicit models, but it turns out to be difficult to use in implicit models. The main difficulty is its nondifferentiable properties associated with an on/off behavior depending on the local vertical density gradient. Local convective adjustment may lead to spurious equilibria (Vellinga 1998) while the global adjustment procedure (Dijkstra et al. 2001) cannot be used in a continuation setup. In most of the results below we will not apply any convective adjustment scheme.

The resulting solutions appear "less realistic" than those of other ocean models with a comparable resolution. However, they are the most "clean" solutions of the governing system of hydrostatic partial differential equations in that boundary layers are well-resolved and no ad hoc procedures for convection have been applied. As will be shown below, the ocean flow is more sluggish, but many characteristics are similar to equilibrium solutions of traditional low-resolution ocean models (with the same spatial resolution).

\section{Results}

Starting from the trivial state, at zero solar forcing, no freshwater flux and no wind stress, first a branch of equilibrium states is computed toward "realistic" forcing conditions. The latter consist of the annual-mean wind stress as in Trenberth et al. (1989), an analytical form of the solar forcing as in the appendix, and the Levitus surface salinity distribution (Levitus et al. 1994). The freshwater flux of this reference solution is diagnosed and subsequently used as part of the surface buoyancy forcing. Next, a steady perturbation pattern in the freshwater flux over a relatively small domain in the North Atlantic is applied and the changes of the equilibrium flows with the amplitude of this perturbation are computed.

\section{a. The unique regime}

For the computation of the reference solution, a restoring time scale of 75 days is used for the Levitus surface salinity. Standard values of eddy diffusivities are $K_{V}=8 \times 10^{-5} \mathrm{~m}^{2} \mathrm{~s}^{-1}$ and $K_{H}=10^{3} \mathrm{~m}^{2} \mathrm{~s}^{-1}$ and the vertical eddy viscosity $A_{V}=10^{-3} \mathrm{~m}^{2} \mathrm{~s}^{-1}$. The properties of the reference steady state are plotted in Fig. 2. In the barotropic streamfunction (Fig. 2a), the gyre structure can be seen in each basin together with the Antarctic Circumpolar Current (ACC). The maximum volume transport of the ACC is about $12 \mathrm{~Sv}\left(\mathrm{~Sv} \equiv 10^{6}\right.$ $\left.\mathrm{m}^{3} \mathrm{~s}^{-1}\right)$, the latter due to the relatively large value of $A_{H}$. The maximum of the Atlantic meridional overturning streamfunction (Fig. 2b) is about $11 \mathrm{~Sv}$, which is slightly smaller than in other models (England 1993). One of the factors causing the smaller overturning is the absence of convective adjustment. The meridional overturning in the Pacific Ocean (Fig. 2c) and the Indian Ocean (Fig. 2d) is relatively strong with extremes of about -12 and $-5 \mathrm{~Sv}$, respectively. In all three oceans, there are Ekman driven surface circulation cells having a relatively small amplitude of a few Sverdrups. In the global overturning streamfunction, the ACC can 

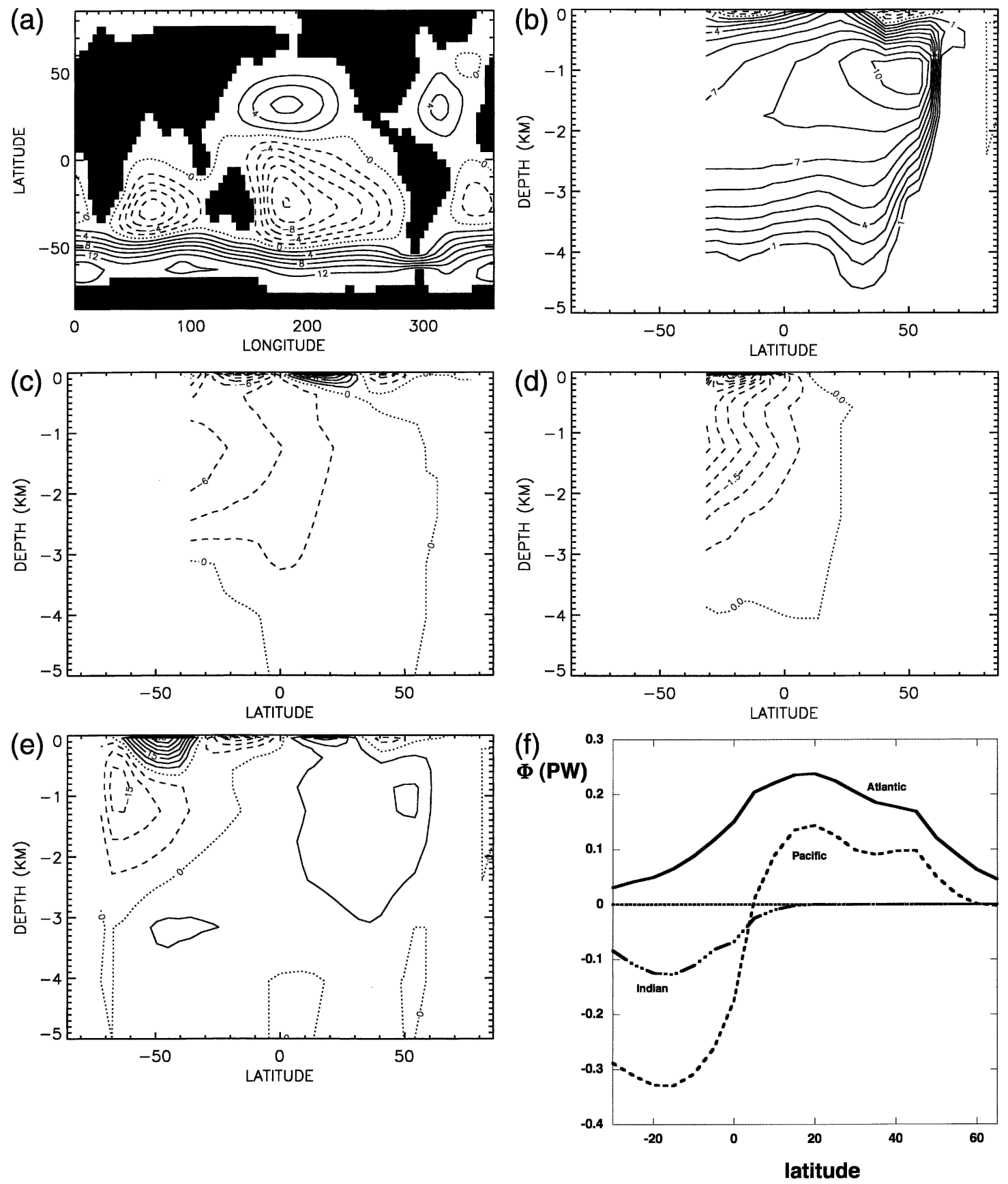

FIG. 2. Properties of the steady global ocean circulation pattern at standard parameter values and forcing conditions, with the surface salinity restored to Levitus values with a time scale of 75 days. (a) Barotropic streamfunction $\left(\Psi_{\min }=\right.$ $\left.-15.8 \mathrm{~Sv}, \Psi_{\max }=12.1 \mathrm{~Sv}\right)$, and meridional overturning streamfunctions in the (b) Atlantic $\left(\Psi_{\min }=-2.9 \mathrm{~Sv}, \Psi_{\max }\right.$ $=10.7 \mathrm{~Sv})$, (c) Pacific $\left(\Psi_{\min }=-12.3 \mathrm{~Sv}, \Psi_{\max }=11.4 \mathrm{~Sv}\right)$, and $(\mathrm{d})$ Indian Ocean $\left(\Psi_{\min }=-5.0 \mathrm{~Sv}, \Psi_{\max }=2.0 \mathrm{~Sv}\right)$. (e) Global ocean $\left(\Psi_{\min }=-18.6 \mathrm{~Sv}, \Psi_{\max }=39.1 \mathrm{~Sv}\right)$. (f) Northward heat transport $(\mathrm{PW})$ over the different basins vs latitude.

be noticed (Fig. 2e) and also the strong southern sinking occurring south of $30^{\circ} \mathrm{S}$ can be seen. The meridional heat transport in the different basins is plotted in Fig. 2f. Qualitatively, the heat transport is correct with northward heat transport over the whole Atlantic, the heat transport in the Pacific changing sign near the equator, and only southward heat transport in the Indian Ocean. The amplitude, however, is a factor of 6 smaller than in observations (Ganachaud and Wunsch 2000). 

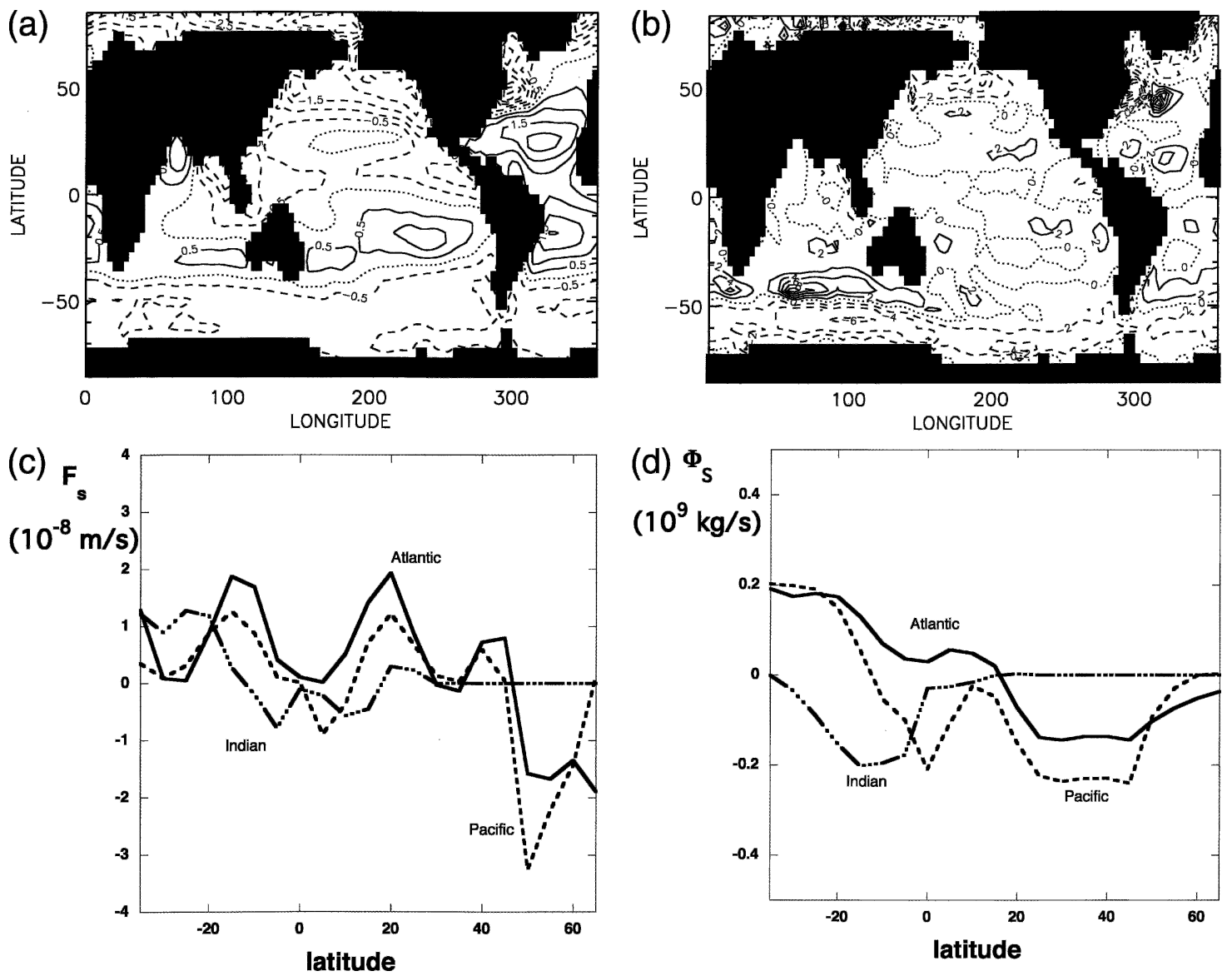

FIG. 3. (a) Surface salinity anomaly with respect to $S_{0}=35 \mathrm{psu}$, with $S_{\min }=-4.7 \mathrm{psu}$ and $S_{\max }=2.2$ psu. (b) Diagnosed freshwater flux field (in units of $10^{-8} \mathrm{~m} \mathrm{~s}^{-1}$ ) of the state in Fig. 2. The minimum and maximum values are $-15.8 \times 10^{-8}$ $\mathrm{m} \mathrm{s}^{-1}$ and $12.5 \times 10^{-8} \mathrm{~m} \mathrm{~s}^{-1}$, respectively. (c) Basin averaged freshwater flux. (d) Northward freshwater transport.

Although many details of this equilibrium solution are incorrect when compared with observations, the overall characteristics of the flow are adequate and, apart from the weaker barotropic transport, comparable to those in other global OGCMs (Manabe and Stouffer 1988; Tziperman et al. 1994; Rahmstorf 1995) using the same (very coarse) resolution. For example, in Manabe and Stouffer (1988), a meridional heat transport of about $0.5 \mathrm{PW}$ is found at $24^{\circ} \mathrm{N}$ in the Atlantic and other models find similar values. The flow here is simply more sluggish than in the other models because of the larger value of $A_{H}$ and the absence of convective adjustment. This leads to the smaller values of the heat transport found. Apart from this, many characteristics (such as the heat transport) are qualitatively correct.

Next, the freshwater flux is diagnosed from this reference solution; this flux (Fig. 3b) is needed to maintain the (interpolated) Levitus surface salt field (shown in Fig. 3a) with the steady state reference flow. Largest amplitudes, with typical values of $3.8 \mathrm{~m} \mathrm{yr}^{-1}$, occur over the southern Indian Ocean and in the northern North Atlantic. Although the freshwater flux differs substantially from the observation based annual mean field
(Oberhuber 1988), the pattern is just as noisy as those diagnosed in other OGCMs using the same resolution (Tziperman et al. 1994) and the large amplitude features roughly correspond. For example, in the North Atlantic, there is a zonal dipolar structure with large amplitude that can also be seen in Fig. 2 of Tziperman et al. (1994). The salt input in the positive part of this dipole near $40^{\circ} \mathrm{N}$ is needed to compensate for the model's incorrect representation of the salt transport due to the Gulf Stream.

In Weaver and Hughes (1996), the same dipolar structure in the freshwater flux is found near other western boundary currents, such as the Kuroshio and Agulhas. These dipoles are neither found here nor in Tziperman et al. (1994). The reason for this difference is probably the higher horizontal resolution (about $2^{\circ}$ ) in the Weaver and Hughes (1996) model. In the interpolation of the Levitus salinity field in the present model, there is hardly any zonal salt gradient in the Kuroshio region. On a higher resolution, this would be the case and hence a compensating effect of the freshwater flux, because of the misrepresentation of the Kuroshio salt transport (as in the Gulf Stream), is required. 

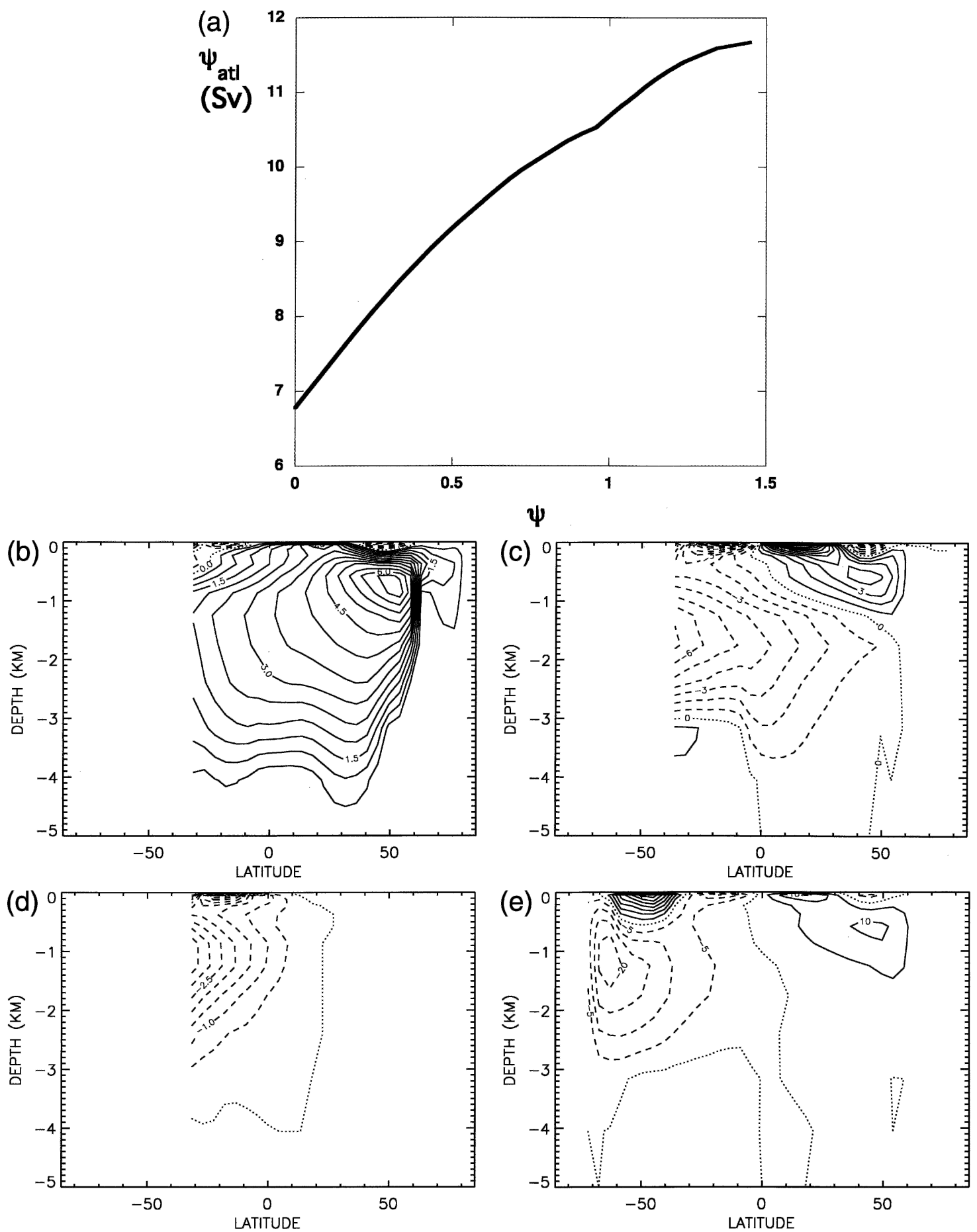

Fig. 4. (a) Bifurcation diagram showing the Atlantic overturning vs the amplitude of the freshwater flux $\gamma$ as in (12). Properties of the solution for $\gamma=0.0$ are plotted with meridional overturning streamfunctions in the (b) Atlantic ( $\left(\Psi_{\text {min }}\right.$ $\left.=-1.9 \mathrm{~Sv}, \Psi_{\max }=6.8 \mathrm{~Sv}\right)$, (c) Pacific $\left(\Psi_{\min }=-9.7 \mathrm{~Sv}, \Psi_{\max }=11.5 \mathrm{~Sv}\right),(\mathrm{d})$ Indian Ocean $\left(\Psi_{\min }=-4.3 \mathrm{~Sv}, \Psi_{\max }=\right.$ $1.5 \mathrm{~Sv})$, and (e) global ocean $\left(\Psi_{\min }=-23.5 \mathrm{~Sv}, \Psi_{\max }=37.8 \mathrm{~Sv}\right)$.

The zonally averaged freshwater fluxes $F_{S}=E-P$ over the different basins have less noise and have the correct order of magnitude (Fig. 3c). Both in the Pacific and the Atlantic, the evaporation regions in the sub- tropics are found with a region of small $F_{S}$ near the equator. The large negative freshwater flux in the north Pacific and Atlantic are needed to provide enough freshwater to maintain the low salinity values in the 
Levitus dataset (Fig. 3a). The meridional freshwater transport in the different basins is shown in Fig. $3 \mathrm{~d}$. Although these are not well known from observations, the values here are about a factor of 6 smaller than current estimates (Wijffels et al. 1992). Although the sign of the freshwater transport is correct in the North Atlantic, it is not in the North Pacific. These model errors are well known from traditional OGCMs and have to be accepted at this low resolution.

In many previous bifurcation studies of thermohaline driven flows [see chapter 6 in Dijkstra (2000)], the structure of steady solutions was investigated with the strength of the freshwater flux as control parameter, while keeping its pattern fixed. If the freshwater flux field in Fig. $3 \mathrm{~b}$ is indicated by $F_{S}^{1}(\phi, \theta)$, then the surface freshwater flux $F_{S}$ is prescribed as

$$
F_{S}=\gamma F_{S}^{1}(\phi, \theta)
$$

where $\gamma$ is a dimensionless amplitude. The bifurcation diagram showing the maximum of the Atlantic meridional overturning streamfunction $\psi_{\text {atl }}$ versus $\gamma$ is plotted in Fig. 4. The value of $\psi_{\text {atl }}$ increases monotonically with $\gamma$ from about $6.8 \mathrm{~Sv}$ at $\gamma=0.0$ up to about $11.5 \mathrm{~Sv}$ at $\gamma=1.5$.

Properties of the thermally only driven solution (for $\gamma=0.0)$ are shown in the Figs. $4 \mathrm{~b}-\mathrm{e}$. Note that for this solution, the salinity is homogeneous over the global ocean. In Fig. 2b, about $75 \%$ of the North Atlantic Deep Water leaves the Atlantic at the southern boundary. This amount has decreased to about $30 \%$ in Fig. 4b as nearly all the deep water reaches the surface before $30^{\circ} \mathrm{S}$. The overturning extends slightly more northward than that of the reference solution. This is because of the freshwater input in the northern North Atlantic (Fig. 3b) present in the forcing of the reference solution.

The southern overturning cell in the Pacific (Fig. 4c) becomes slightly weaker (it has changed to $-9.7 \mathrm{~Sv}$ ), and there is now even a northern overturning cell of about $4 \mathrm{~Sv}$. The strong freshwater input in the North Pacific (Fig. 3b) inhibits the presence of this northern overturning cell in the reference solution. The overturning streamfunction in the Indian Ocean (Fig. 4d) and the global barotropic streamfunction (not shown) are hardly affected. In the global meridional overturning streamfunction (Fig. 4e) the amplitude of the southern sinking cell has increased to about $20 \mathrm{~Sv}$.

It is clear that each equilibrium of the global ocean circulation is unique for a fixed value of $\gamma$ (Fig. 4a). If a transient simulation would be started from the reference equilibrium solution $(\gamma=1)$ it would remain near that state under the prescribed freshwater flux, similar to the large $\tau_{S}$ results in Tziperman et al. (1994).

\section{b. The multiple-equilibria regime}

We first define a region near Newfoundland, similar to region A in Rahmstorf (1995), with domain $(\phi, \theta) \in$
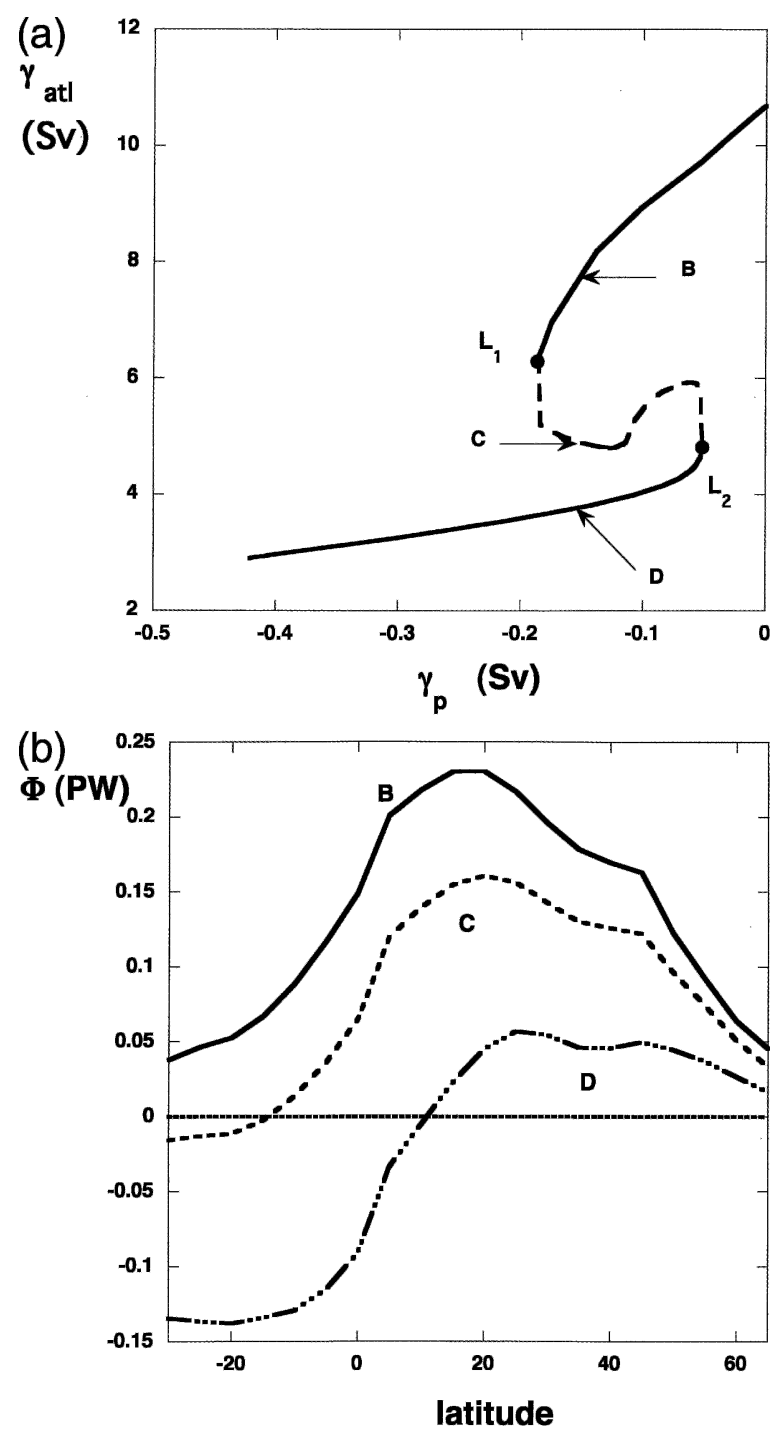

FIG. 5. (a) Bifurcation diagram of the steady states of the global ocean circulation as a plot of the maximum meridional Atlantic overturning vs the anomalous freshwater-flux strength $\gamma_{p}(\mathrm{~Sv})$. Note that for $\gamma_{p}=0$, the reference solution is obtained. (b) Changes in the Atlantic meridional heat transport along the curve in (a).

$\left[60^{\circ}-24^{\circ} \mathrm{W}\right] \times\left[54^{\circ}-66^{\circ} \mathrm{N}\right]$. To study the impact of changes in the freshwater flux pattern, a perturbation flux $F_{S}^{p}$ is defined as

$$
F_{S}^{p}=\gamma_{p} F_{S}^{2}(\phi, \theta)
$$

where $F_{S}^{2}(\phi, \theta)=1$ in the region near Newfoundland and zero outside. The value of $\gamma_{p}$ controls the amplitude of the freshwater flux perturbation, and following previous model studies, it will be expressed in Sverdrups. When changing $\gamma_{p}$, one has to take care that the salt balance is closed; that is, we subtract the surfaceintegrated value $Q$ from the total freshwater flux profile, such that 

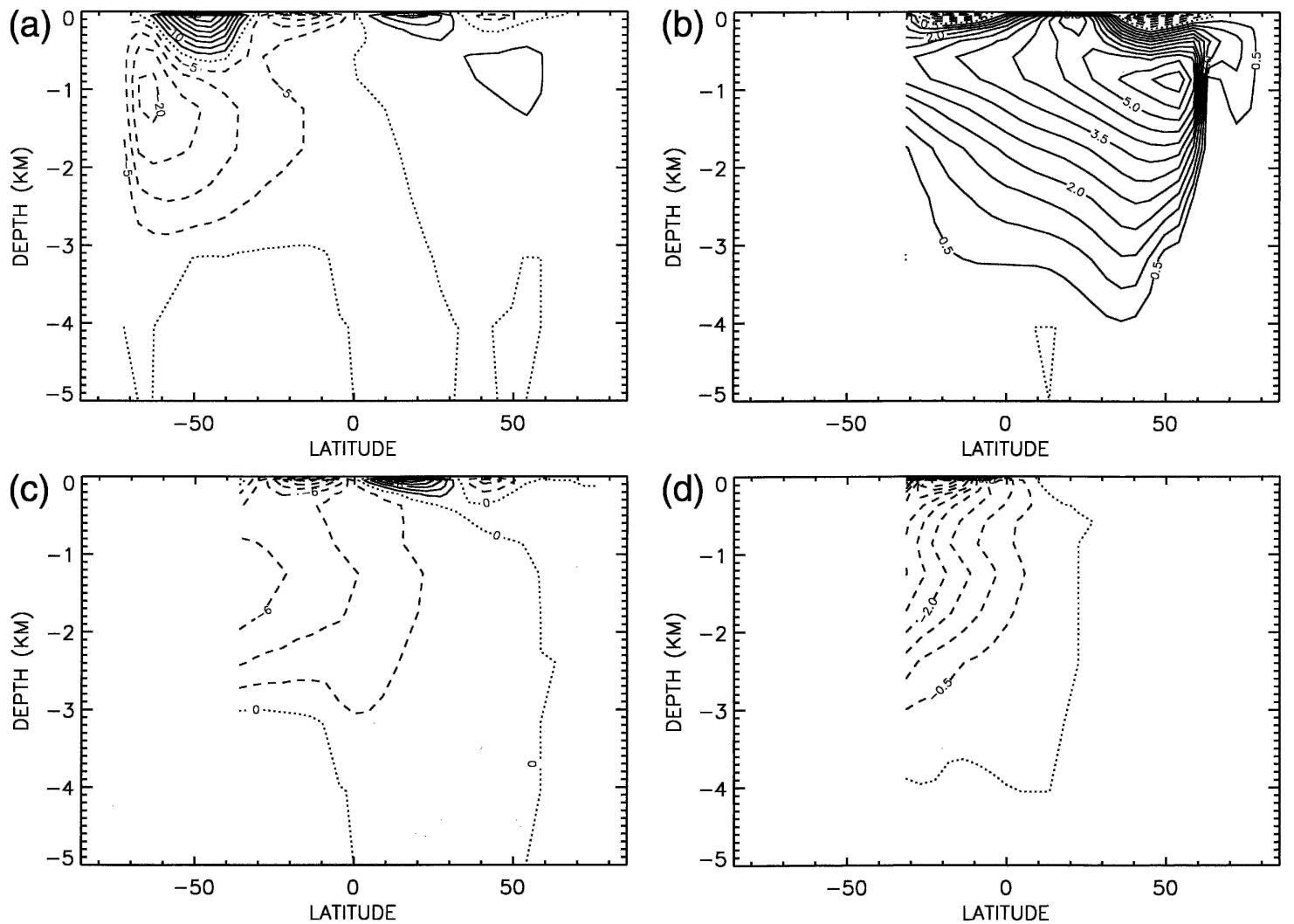

FIG. 6. Properties of the steady global ocean circulation pattern at the saddle-node bifurcation $\mathrm{L}_{1}$ in Fig. 5. Meridional overturning streamfunction: (a) global ocean $\left(\Psi_{\min }=-22.1 \mathrm{~Sv}, \Psi_{\max }=38.5 \mathrm{~Sv}\right)$, (b) Atlantic $\left(\Psi_{\min }=-2.7 \mathrm{~Sv}, \Psi_{\max }=\right.$ 6.4 Sv), (c) Pacific $\left(\Psi_{\min }=-12.4 \mathrm{~Sv}, \Psi_{\max }=11.5 \mathrm{~Sv}\right)$, and (d) Indian Ocean $\left(\Psi_{\min }=-5.1 \mathrm{~Sv}, \Psi_{\max }=1.9 \mathrm{~Sv}\right)$.

$\frac{1}{|S|} \int_{S}\left[F_{S}^{1}(\phi, \theta)+\gamma_{p} F_{S}^{2}(\phi, \theta)\right] r_{0}^{2} \cos \theta d \theta d \phi-Q=0$,

where $|S|$ is the total area of the ocean surface.

The bifurcation diagram, showing the maximum Atlantic meridional overturning of the steady solutions versus $\gamma_{p}$ is plotted in Fig. 5a. It consists of two saddlenode bifurcations, indicated by $\mathrm{L}_{1}\left(\gamma_{p}=-0.18 \mathrm{~Sv}\right)$ and $\mathrm{L}_{2}\left(\gamma_{p}=-0.052 \mathrm{~Sv}\right)$, that exactly bound the regime of multiple equilibria of these flows. Stable solutions are indicated by a continuous line style while the branch of unstable solutions is dashed. From the reference solution $\left(\gamma_{p}=0.0\right)$ in this model configuration (Fig. 2), one needs to increase the amplitude of the freshwater flux perturbation beyond $\gamma_{p}=-0.052 \mathrm{~Sv}$ to reach into the multiple-equilibria regime. In this regime, a transition to a weak Atlantic MOC state can occur. For values smaller than $\gamma_{p}=-0.18 \mathrm{~Sv}$, only the latter state exists.

The changes in the Atlantic meridional heat transport along the equilibrium curve are plotted in Fig. 5b. The labels refer to solutions marked at specific locations in Fig. 5a. At point B, the heat transport is still positive over the Atlantic, but along the unstable branch (point C) the heat transport changes sign in the South Atlantic. In the weak MOC state, the heat transport is qualitatively similar to that Pacific in the reference solution (Fig. 2f). This behavior of the heat transport is consistent with the decrease in meridional overturning as found in Fig. 5a.

With decreasing $\gamma_{p}$, starting from the reference solution, the Atlantic overturning decreases until the saddle-node bifurcation $\mathrm{L}_{1}$ at $\gamma_{p}=-0.18 \mathrm{~Sv}$. Note that the equilibrium states on this branch are still linearly stable in the multiple equilibrium regime $(-0.18 \mathrm{~Sv}<$ $\left.\gamma_{p}<-0.052 \mathrm{~Sv}\right)$. Hence, when a transient computation is started from an initial condition that is only a small perturbation of the equilibrium state (e.g., by a small change in the freshwater input over a certain region), it will return to the equilibrium state. A finite-amplitude perturbation is therefore needed to induce a transition to the stable weak MOC state on the lower branch in Fig. 5a and whether a transition will occur depends on the amplitude of this perturbation. Note that, because the steady states change with $\gamma_{p}$, also the critical amplitude of this finite-amplitude perturbation changes with $\gamma_{p}$.

The solution near the saddle-node bifurcation $\mathrm{L}_{1}$ in Fig. 5a is shown in Fig. 6. The surface salinity field in the Atlantic (not shown) has decreased by about 1 psu 

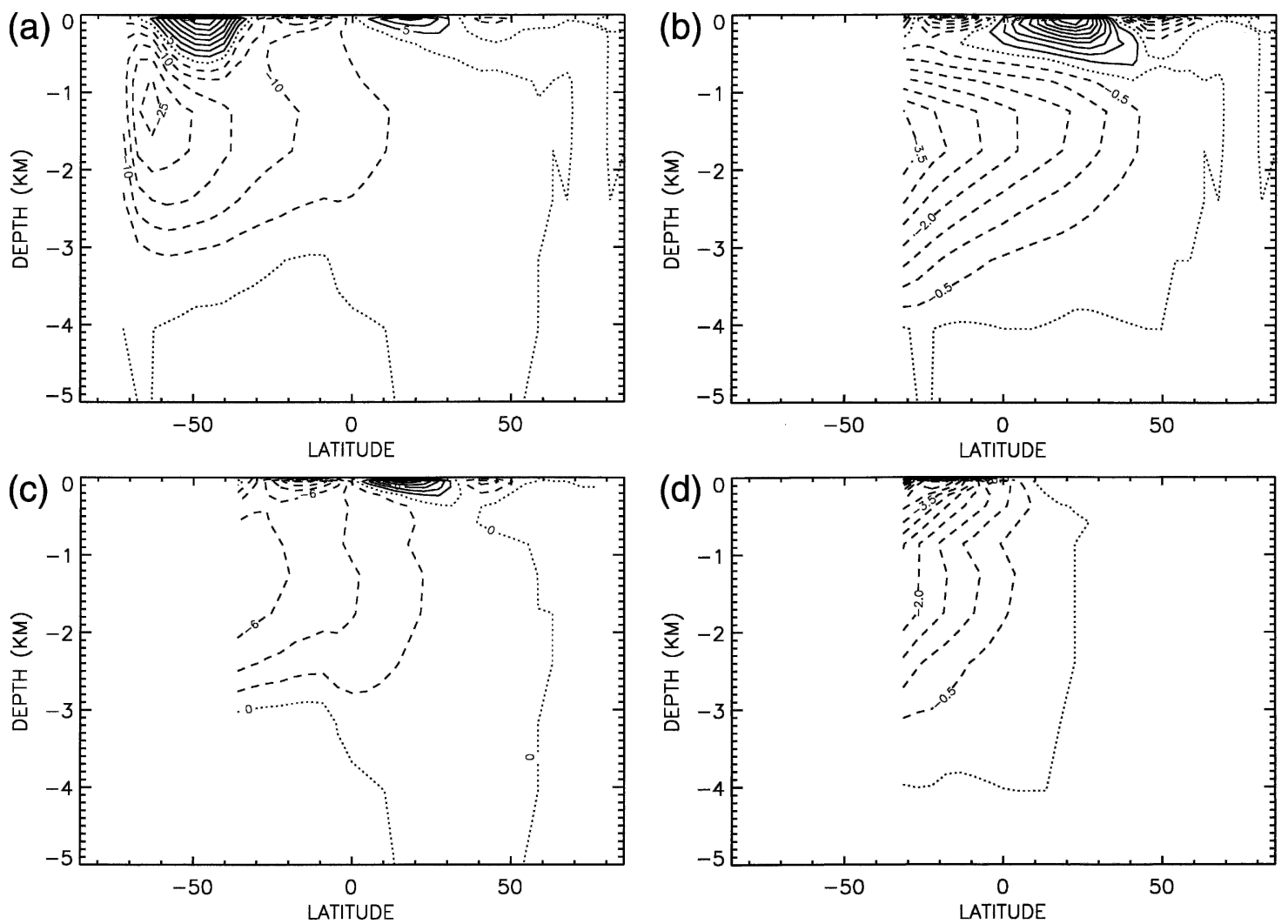

FIG. 7. Properties of the steady global ocean circulation pattern at the saddle-node bifurcation $\mathrm{L}_{2}$ in Fig. 5. Meridional overturning streamfunction: (a) global $\left(\Psi_{\min }=-27.3 \mathrm{~Sv}, \Psi_{\max }=37.6 \mathrm{~Sv}\right)$, (b) Atlantic $\left(\Psi_{\min }=-3.9 \mathrm{~Sv}, \Psi_{\max }=5.0 \mathrm{~Sv}\right)$, (c) Pacific $\left(\Psi_{\min }=-12.4 \mathrm{~Sv}, \Psi_{\max }=11.4 \mathrm{~Sv}\right)$, and (d) Indian Ocean $\left(\Psi_{\min }=-5.1 \mathrm{~Sv}, \Psi_{\max }=1.8 \mathrm{~Sv}\right)$.

with respect to that of the reference solution. In the global overturning streamfunction (Fig. 6a), the southern overturning in the region south of the $30^{\circ}$ has increased with respect to that of the reference solution and the northern overturning in the Northern Hemisphere has weakened. Only the changes in the Atlantic overturning (Fig. 6b) contribute to this (it has weakened) as the overturning circulation in the other basins is hardly affected (Figs. 6b,c). This indicates a fairly localized response of the equilibrium circulation to the local anomalous freshwater flux. In the North Atlantic, the latitude of sinking has slightly moved southward with respect to the reference solution and there is a small amount of bottom water at depth. The upwelling of deep water has also increased since the streamlines in (Fig. 6a) are tilted upward to the south.

Along the unstable (dashed) branch in Fig. 5a, the Atlantic overturning strength of the northern sinking solution seems to remain fairly constant. However, this is a consequence of the norm chosen since it is determined by the wind-driven Ekman cell at the surface. Actually, it is along this branch that the solution totally changes character: northern sinking is inhibited and the volume of bottom water coming from the south increases. Last, the northern sinking cell in the Atlantic has basically disappeared, while the southern sinking cell has increased in amplitude to an overturning strength of about $-4 \mathrm{~Sv}$ at the saddle-node bifurcation $\mathrm{L}_{2}$ at $\gamma_{p}=-0.052 \mathrm{~Sv}$.

The solution at this saddle-node bifurcation is shown in Fig. 7. There is only a slight region in the subtropical Atlantic where the salinity is larger than 35 psu. The sinking in the Southern Hemisphere has increased by 5 Sv (Fig. 7a), but the pattern of the global overturning is fairly similar to that in Fig. 6a. There is still a small subsurface sinking with a maximum of about $5 \mathrm{~Sv}$ in the North Atlantic (Fig. 7b) but, otherwise, sinking only occurs in the Southern Ocean. Again, the whole reorganization of the Atlantic overturning pattern has hardly any influence on the flows in the Pacific and Indian Ocean (Figs. 7c,d). The maximum Indian Ocean meridional overturning has only changed by about $1 \%$ along the whole unstable branch (and the maximum Pacific overturning even less).

Along the lower branch (lower continuous line segment in Fig. 5a), the Atlantic overturning decreases with increasing freshwater input (more negative $\gamma_{p}$ ). The solution in Fig. 8 shows the southern sinking (weak MOC) state at a quite large negative value of $\gamma_{p}=$ $-0.42 \mathrm{~Sv}$. The sea surface salinity field now shows a 

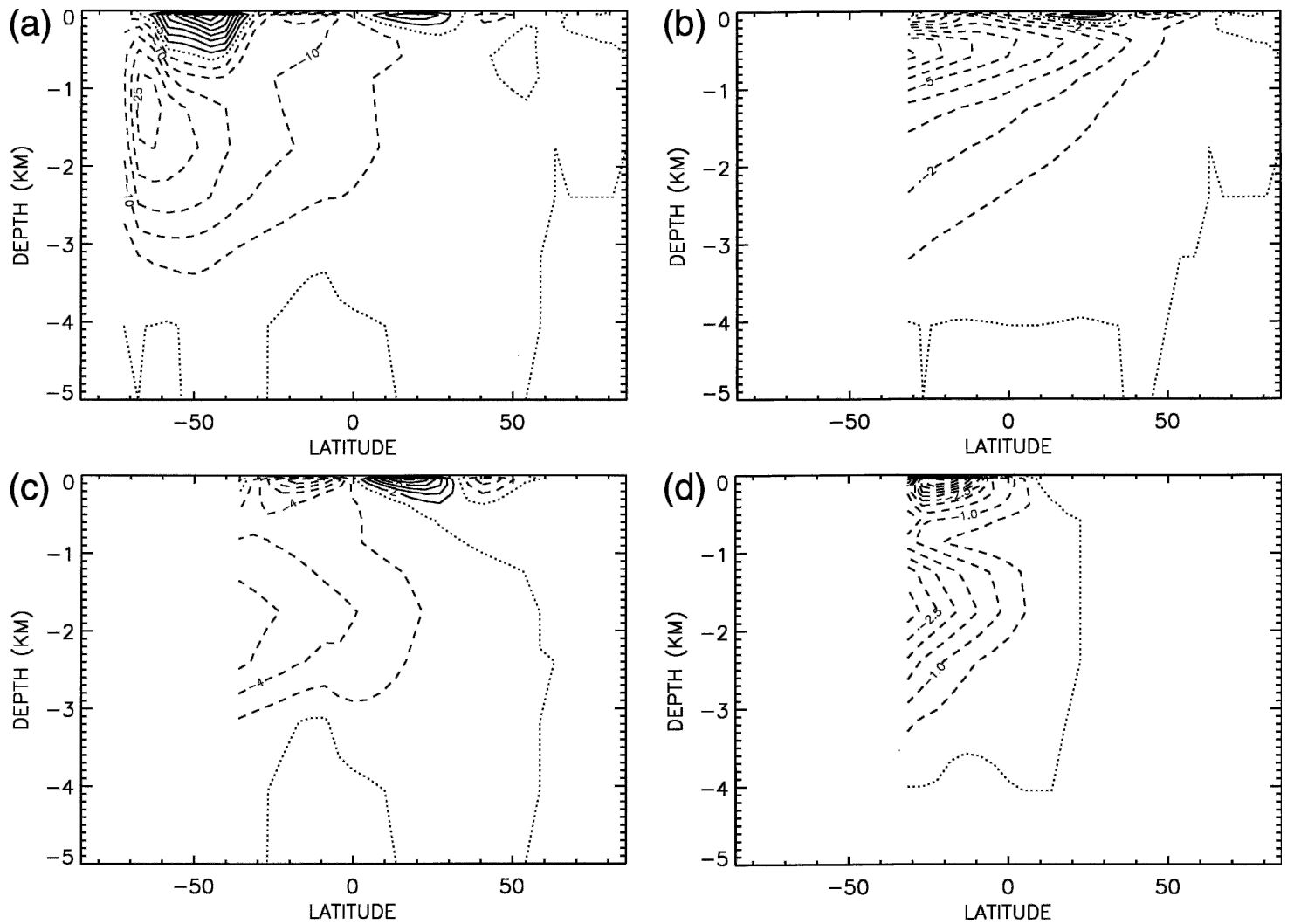

FIG. 8. Properties of the steady global ocean circulation pattern at the left endpoint of the branch in Fig. 5 at $\gamma_{p}=-0.42$ Sv. Meridional overturning streamfunction: (a) global ocean $\left(\Psi_{\min }=-28.7 \mathrm{~Sv}, \Psi_{\max }=36.1 \mathrm{~Sv}\right)$, (b) Atlantic $\left(\Psi_{\min }=\right.$ $\left.-8.3 \mathrm{~Sv}, \Psi_{\max }=2.9 \mathrm{~Sv}\right)$, (c) Pacific $\left(\Psi_{\min }=-12.0 \mathrm{~Sv}, \Psi_{\max }=12.4 \mathrm{~Sv}\right)$, and (d) Indian Ocean $\left(\Psi_{\min }=-5.7 \mathrm{~Sv}, \Psi_{\max }\right.$ $=1.3 \mathrm{~Sv})$.

very strong negative anomaly of about $10 \mathrm{psu}$ in the North Atlantic and in nearly all of the Atlantic, the salinity is lower than 35 psu. While the global meridional overturning has not changed much (Fig. 8a), the maximum of southern overturning in the Atlantic has shifted upward such that the Ekman cells are now hardly visible (Fig. 8b). In the Pacific and Indian Ocean, the salinity has increased because of the constraint (14). It is due to these changes in the salinity field that the overturning in Pacific and Indian Ocean (Figs. 8c,d) is also affected and both show a $10 \%$ increase in southern overturning with respect to the solution at $\mathrm{L}_{2}$.

\section{c. Sensitivity to $K_{V}$}

From studies with global ocean models, it is found that the hysteresis behavior depends on the vertical mixing coefficient. For example, Prahl et al. (2003) find, by varying the surface values of the vertical mixing coefficient $K_{V}$, that the hysteresis does exist over a large range of $K_{V}$. For small values of $K_{V}$ the hysteresis interval decreases with decreasing values of $K_{V}$ and it finally seems to disappear.

In the quasi-equilibrium computations with OGCMs, the multiple-equilibria regime has to be estimated from the hysteresis loop. This is not trivial since, even before the saddle-node bifurcation is reached, the equilibrium solution already reacts to the quasi-steady freshwater changes. Complementary to the quasi-equilibrium computations, the results here provide the exact location of the saddle-node bifurcations $\left(\mathrm{L}_{1}\right.$ and $\mathrm{L}_{2}$ in Fig. 5a). Hence, by following the $\gamma_{p}$ position of the saddle-node bifurcations in $K_{V}$, a regime diagram in the twoparameter $\left(\gamma_{p}, K_{V}\right)$ plane is obtained. This regime diagram exactly shows the sensitivity of the multipleequilibria regime to $K_{V}$.

Bifurcation diagrams for the smallest and largest values of $K_{V}$ used are plotted in Fig. 9a. The topological shape of the diagrams is similar and for both cases, the double saddle-node structure is found. The distance in $\gamma_{p}$ between the saddle-node bifurcations decreases slightly with $K_{V}$, as can also be seen in the regime diagram plotted in Fig. $9 \mathrm{~b}$ over the range of $8.0 \times 10^{-5}<$ $K_{V}<10^{-3} \mathrm{~m}^{2} \mathrm{~s}^{-1}$. Note that the fact that one of the saddle-node bifurcations for $K_{V}=10^{-3}$ is located on the curve for $K_{V}=8 \times 10^{-5}$ does not mean anything; it is just because of the choice of norm.

Our results are qualitatively similar to those in Prahl et al. (2003) although the procedure used is slightly different. While they use the same freshwater flux for 

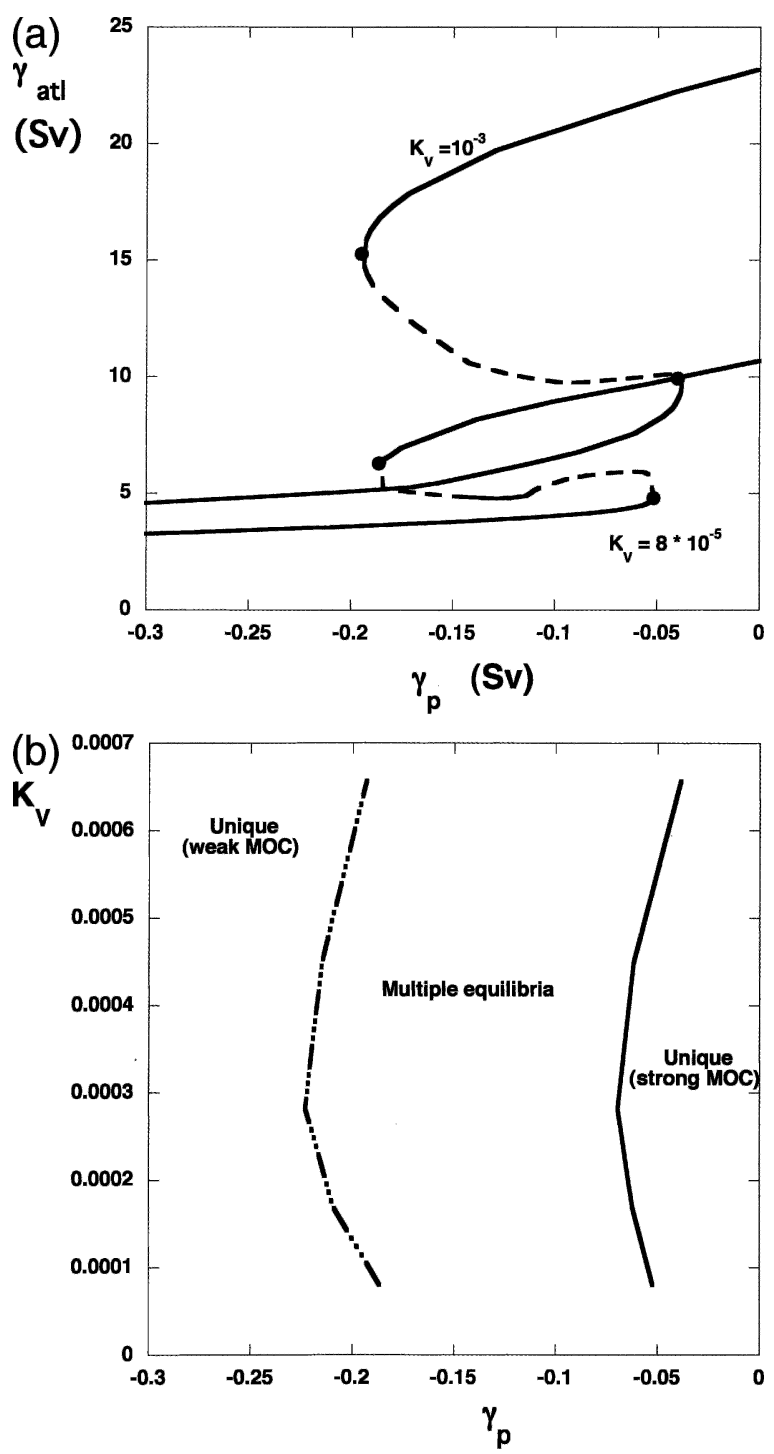

FIG. 9. (a) Bifurcation diagrams for two values of $K_{V}$ as curves of the maximum Atlantic meridional overturning vs the strength of the anomalous freshwater flux $\gamma_{p}$. (b) Regime diagram in the two-parameter $\left(\gamma_{p}, K_{V}\right)$ plane.

each value of $K_{V}$, we use the diagnosed freshwater flux obtained from the restoring solution at each value of $K_{V}$. Hence, in our case, the diagnosed flux is able to maintain the Levitus surface salinity distribution at $\gamma_{p}$ $=0$. The fact that no hysteresis is found at very small values of $K_{V}$ in Prahl et al. (2003) is probably due to fact that the freshwater flux they use can only sustain the weak MOC solution (for all values of $\gamma_{p}$ ) and hence a unique solution exists.

\section{Summary and discussion}

For the first time, explicit bifurcation diagrams of the global ocean circulation have been calculated for a low- resolution OGCM by techniques from dynamical systems theory. For a configuration and parameter setup chosen quite similar to those in England (1993) and Rahmstorf (1995) except for the large lateral viscosity, which makes the flow more sluggish, steady ocean circulation patterns are determined in parameter space. Without the computation of a single transient flow, both unique and multiple-equilibria regimes are found. For the standard configuration, it turns out that there are no multiple equilibria of the global ocean circulation under a freshwater flux, which is diagnosed from the Levitus surface salinity field. Multiple equilibria only appear when the northern North Atlantic Ocean is freshened. In that case, in addition to the northern sinking equilibrium, a solution with southern sinking is also dynamically possible.

Previous model studies have shown indications that the location of the hysteresis regime depends on various processes, such as vertical mixing (Manabe and Stouffer, 1999) and the restoring time scale of salinity in the reference solution of Tziperman et al. (1994), which are assumed constant in the present study. The techniques to compute equilibria of large-dimensional dynamical systems, here with a dimension of about 280000 , allow us to determine systematically the sensitivity of the global ocean circulation to the strength and representation of the various large-scale processes. Although this work is still in progress, some scenarios can already be anticipated.

For example, because of the different restoring time scale for salinity in the results by Tziperman et al. (1994) different equilibrium points within the diagram are reached. Their equilibrium computed with the smaller value of $\tau_{S}$ experiences an additional freshwater flux into the North Atlantic as compared with their reference solution. A decreasing value of $\tau_{S}$ is therefore mimicked in the model here as a more negative value of $\gamma_{p}$. Indeed, the latter promotes the multiple-equilibria regime as is seen in Fig. 5a. While for large $\tau_{S}$, it is difficult to determine whether the equilibrium is in the unique or multiple-equilibria regime, the solution for small $\tau_{S}$ is certainly in the multiple-equilibria regime because it is unstable according to Tziperman et al. (1994); hence it must be located on the unstable branch. A similar view may apply to the results of Manabe and Stouffer (1999) where a larger value of the vertical mixing coefficient of heat and salt likely shifts the ocean state from the multiple equilibria into the unique regime.

The existence of the multiple-equilibria regime is not critically dependent on convective adjustment, since it is here also found in a model in which this procedure is not applied. Omitting this procedure has lead to a smooth single branch in Fig. 5a instead of the highly variable temporal signature as found in quasiequilibrium simulations (Rahmstorf 1995). Convective adjustment makes the equilibria very sensitive to local finite-amplitude perturbations, which results in excur- 
(a)

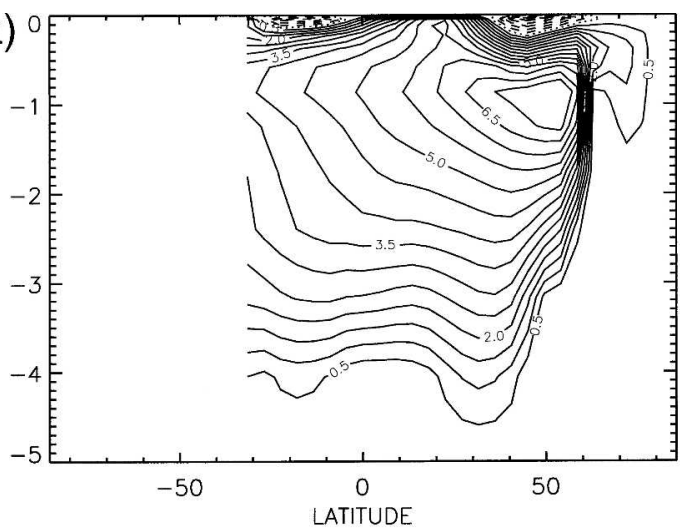

(c)

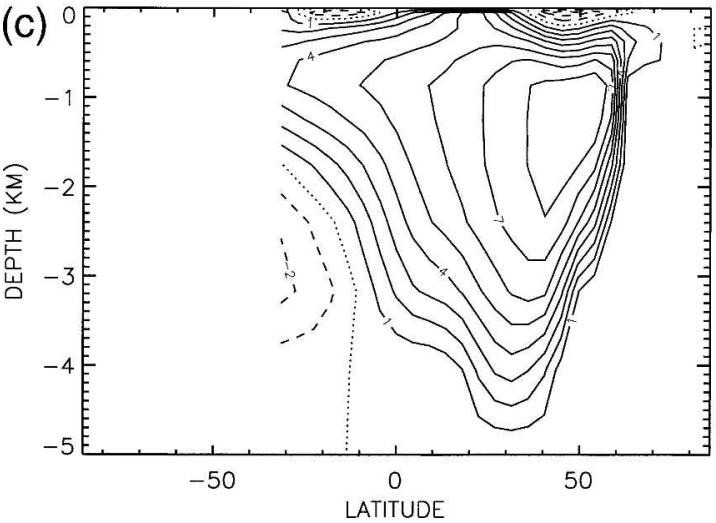

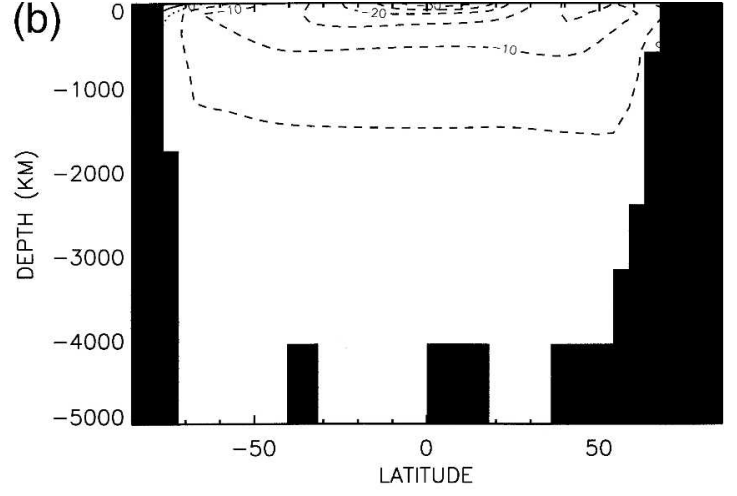

(d)

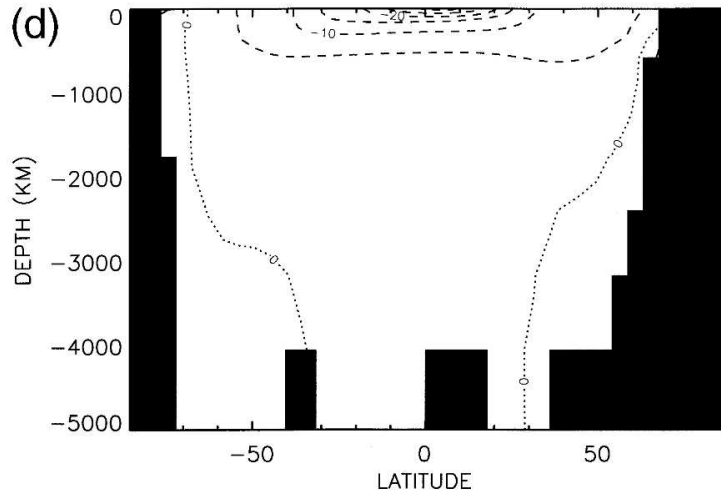

FIG. 10. Comparison of the unadjusted state corresponding to point B in Fig. 5a and the adjusted state for the same parameter values. (a) Atlantic meridional overturning streamfunction $\left(\Psi_{\max }=7.8 \mathrm{~Sv}\right)$ of the unadjusted state. (b) Density profile of the unadjusted state. (c) Atlantic meridional overturning streamfunction $\left(\Psi_{\max }=8.9 \mathrm{~Sv}\right)$ of the adjusted state. (d) Density profile of the adjusted state.

sions to nearby local equilibria in a quasi-equilibrium simulation (Vellinga 1998). The multiple-equilibria regime is shown here to be fairly robust to changes in $K_{V}$ similar to results in traditional ocean models (Prahl et al. 2003).

As shown in Dijkstra et al. (2001) for singlehemispheric thermohaline flows, the difference between the unadjusted and adjusted solutions is usually a few Sverdrup, while the overall pattern of the overturning remains the same. In Fig. 10, the Atlantic meridional overturning streamfunction and a density section (at $22.5^{\circ} \mathrm{W}$ ) are plotted for the adjusted and unadjusted flow for the parameters corresponding to point B in Fig. 5 a. Because of the adjustment, which can be clearly seen in the density profiles, the overturning has increased by about $1.5 \mathrm{~Sv}$, and indeed the overturning pattern is not changed much, except for the larger amount of deep water coming from the south. This would indicate that the adjusted state is closer to the saddle-node bifurcation (at $\gamma=1.25$ ) than the unadjusted state.

The locations of the two saddle-node bifurcations in parameter space are crucial to distinguish unique and multiple equilibria regimes. Based on many quasiequilibrium calculations with ocean models, the exis- tence of these bifurcations seems to be robust. However, the quantitative location of these points in parameter space is expected to be model dependent. In the coupled model here, we find that the reference state is in the unique regime, but in the configuration of Rahmstorf (1995), the reference state is in the hysteresis regime. Differences in model setup are the presence of the energy-balance model of the atmosphere, the larger value of the horizontal friction, the use of a different wind stress field, and, maybe, the use of convective adjustment. The advantage of the methodology used here is that the effect of these differences can now be systematically studied.

It turns out that the spatial pattern of the freshwater flux is the sensitive element in the response of the model. Both the diagnosed flux as well as the shape of the perturbation pattern will introduce a shift of the locations of both saddle-node bifurcations in Fig. 5a. Also the impact of other conditions, such as glacial forcing conditions or changes in radiation through increased greenhouse gases, will probably have most effect on the bifurcation diagram through the changes in the freshwater flux pattern. This is worrisome because it introduces a fair degree of quantitative nonrobustness in the response of low-resolution ocean models 
that are often used as components in climate models. The spatial pattern of the freshwater flux is very difficult to determine in the latter models with an atmospheric component that captures only large-scale processes. In model-model intercomparisons, patterns of the freshwater flux will differ widely and hence their corresponding ocean equilibrium solutions, with largest differences in the North Atlantic. This quantitative nonrobustness is certainly undesired in studies assessing the effect of increasing atmospheric greenhouse gas concentrations (Houghton et al. 2001).

Acknowledgments. This work was supported by the Netherlands Organization for Scientific Research (NWO) under a PIONIER grant to HD. We thank one of the anonymous reviewers for the helpful comments on the manuscript, which lead to a much improved paper. All computations were done on the Origin 3800 computer at SARA Amsterdam. Use of these computing facilities was sponsored by the National Computing Facilities Foundation (N.C.F.) under Project SC029 with financial support from NWO.

\section{APPENDIX}

\section{Atmospheric Energy Balance Model}

The atmospheric model used is one of the simplest versions of the class of energy-balance models (North et al. 1981). The freshwater flux is prescribed instead of being determined from a moisture budget equation. The equation for the atmospheric surface temperature $T_{a}$ on the global domain is given by

$$
\begin{aligned}
\rho_{a} H_{a} C_{p a} \frac{\partial T_{a}}{\partial t}= & \rho_{a} H_{a} C_{p a} D_{0} \nabla\left[D(\theta) \nabla T_{a}\right]-\left(A+B T_{a}\right) \\
& +\frac{\Sigma_{0}}{4} S(\theta)(1-\alpha)\left(1-C_{0}\right) \\
& +\mu_{o a}(1-\mathcal{L})\left(T-T_{a}\right)+\mu_{l a} \mathcal{L}\left(T_{l}-T_{a}\right)
\end{aligned}
$$

where $\rho_{a}=1.25 \mathrm{~kg} \mathrm{~m}^{-3}$ is the atmospheric density, $C_{p a}$ $=10^{3} \mathrm{~J}(\mathrm{~kg} \mathrm{~K})^{-1}$ is the heat capacity, $\alpha=0.3$ is the constant albedo, $H_{a}=8.4 \times 10^{3} \mathrm{~m}$ is an atmospheric scale height, $\Sigma_{0}=1.36 \times 10^{3} \mathrm{~W} \mathrm{~m}^{-2}$ is the solar constant, $D_{0}=3.1 \times 10^{6} \mathrm{~m}^{2} \mathrm{~s}^{-1}$ is a constant eddy diffusivity, and $1-C_{0}=0.57$ is the atmospheric absorption coefficient. The functions $D(\theta)$ and $S(\theta)$ give the latitude dependence of the eddy diffusivity and the shortwave radiative heat flux with

$$
\begin{aligned}
& D(\theta)=0.9+1.5 \exp \left(-\frac{12 \theta^{2}}{\pi}\right) \text { and } \\
& S(\theta)=1-\frac{1}{2}\left[0.482(3 \sin \theta)^{2}-1\right] .
\end{aligned}
$$

The constants $A=216 \mathrm{~W} \mathrm{~m}^{-2}$ and $B=1.5 \mathrm{~W} \mathrm{~m}^{-2} \mathrm{~K}^{-1}$ control the magnitude of the longwave radiative flux.
Last, $T$ is the sea surface temperature, $T_{l}$ the temperature of the land surface points, and the coefficient $\mathcal{L}$ indicates whether the underlying surface is ocean $(\mathcal{L}$ $=0)$ or land $(\mathcal{L}=1)$. The exchange of heat between atmosphere and ocean and between atmosphere and the land surface is modeled by constant exchange coefficients. We assume here for simplicity that both are equal, with

$$
\mu_{l a}=\mu_{o a}=\rho_{a} C_{p a} C_{H}\left|V_{a}\right| \equiv \mu,
$$

where $C_{H}=1.22 \times 10^{-3}$ and $\left|V_{a}\right|=8.5 \mathrm{~m} \mathrm{~s}^{-1}$ is a mean atmospheric surface wind speed; it follows that $\mu \approx 13$ $\mathrm{W} \mathrm{m}{ }^{-2} \mathrm{~K}^{-1}$.

Boundary conditions for the atmosphere are periodic in zonal direction and no-flux conditions at the northsouth boundaries; that is,

$$
\begin{aligned}
T_{a}\left(\phi=\phi_{W}\right) & =T_{a}\left(\phi=\phi_{E}\right) ; \\
\frac{\partial T_{a}}{\partial \phi}\left(\phi=\phi_{W}\right) & =\frac{\partial T_{a}}{\partial \phi}\left(\phi=\phi_{E}\right) \\
\theta & =-\theta_{S}, \theta_{N}: \frac{\partial T_{a}}{\partial \theta}=0 .
\end{aligned}
$$

The net downward heat flux into the ocean and land is given by

$$
Q_{o a}=\frac{\Sigma_{0}}{4} S(\theta)(1-\alpha) C_{0}-\mu\left(T-T_{a}\right)
$$

and

$$
Q_{l a}=\frac{\Sigma_{0}}{4} S(\theta)(1-\alpha) C_{0}-\mu\left(T_{l}-T_{a}\right) .
$$

When the heat capacity of the land is assumed to be zero, then $Q_{l a}=0$ and the land temperature $T_{l}$ is computed directly from

$$
T_{l}=T_{a}+\frac{\Sigma_{0}}{4 \mu} S(\theta)(1-\alpha) C_{0} .
$$

In this way, the following integral conditions are satisfied in steady state

$$
\int_{S} Q_{o a}(1-\mathcal{L}) d^{2} x=0
$$

and

$$
\int_{S}\left[\frac{\Sigma_{0}}{4} S(\theta)(1-\alpha)-\left(A+B T_{a}\right)\right] d^{2} x=0,
$$

with the integral

$$
\int_{S} d^{2} x=\int_{\phi_{W}}^{\phi_{E}} \int_{\theta_{S}}^{\theta_{N}} r_{0}^{2} \cos \theta d \phi d \theta .
$$

From the last integral condition it follows that the average atmospheric temperature is independent of the land-sea distribution. 


\section{REFERENCES}

Alley, R. B., P. A. Mayewski, T. Sowers, M. Stuiver, K. C. Taylor, and P. A. Clark, 1997: Holocene climate variability: A prominent widespread event 8200 years ago. Geology, 25, 483-486.

Botta, E. F. F., and F. W. Wubs, 1999: MRILU: An effective algebraic multilevel ILU-preconditioner for sparse matrices. SIAM J. Matrix Anal. Appl., 20, 1007-1026.

Broecker, W. S., 2000: Abrupt climate change: Causal constraints provided by the paleoclimate record. Earth-Sci. Rev., 51, 137154.

Bryan, F. O., 1986: High-latitude salinity effects and interhemispheric thermohaline circulations. Nature, 323, 301-304.

Clark, P. U., N. G. Pisias, T. F. Stocker, and A. J. Weaver, 2002: The role of the thermohaline circulation in abrupt climate change. Nature, 415, 863-869.

Dijkstra, H. A., 2000: Nonlinear Physical Oceanography: A Dynamical Systems Approach to the Large Scale Ocean Circulation and El Niño. Kluwer Academic, 456 pp.

—, and W. Weijer, 2003: Stability of the global ocean circulation: The connection of equilibria in a hierarchy of models. $J$. Mar. Res., 61, 725-743.

— , H. Öksüzŏglu, F. W. Wubs, and E. F. F. Botta, 2001: A fully implicit model of the three-dimensional thermohaline ocean circulation. J. Comput. Phys., 173, 685-715.

— W. Weijer, and J. D. Neelin, 2003: Imperfections of the three-dimensional thermohaline ocean circulation: Hysteresis and unique state regimes. J. Phys. Oceanogr., 33, 27962814.

England, M. H., 1993: Representing the global-scale water masses in ocean general circulations models. J. Phys. Oceanogr., 23, $1523-1552$.

Ganachaud, A., and C. Wunsch, 2000: Improved estimates of global ocean circulation, heat transport and mixing from hydrographic data. Nature, 408, 453-457.

Gordon, A. L., 1986: Interocean exchange of thermocline water. $J$. Geophys. Res., 91, 5037-5046.

Hall, M., and H. Bryden, 1982: Direct estimates of ocean heat transport. Deep-Sea Res., 29, 339-359.

Houghton, J. T., Y. Ding, D. J. Griggs, M. Noguer, P. J. van der Linden, X. Dai, K. Maskell, and C. A. Johnson, Eds., 2001: Climate Change 2001: The Scientific Basis. Cambridge University Press, $881 \mathrm{pp}$.

Keller, H. B., 1977: Numerical solution of bifurcation and nonlinear eigenvalue problems. Applications of Bifurcation Theory, P. H. Rabinowitz, Ed., Academic Press, 359-385.

Levitus, S., R. Burgett, and T. Boyer, 1994: Salinity. Vol. 3, World Ocean Atlas 1994, NOAA Atlas NESDIS 3, 99 pp.

Manabe, S., and R. J. Stouffer, 1988: Two stable equilibria of a coupled ocean-atmosphere model. J. Climate, 1, 841-866.

- and - 1999: Are two modes of thermohaline circulation stable? Tellus, 51A, 400-411.

North, G. R., R. F. Cahalan, and J. A. Coakley, 1981: Energy balance climate models. Rev. Geophys. Space Phys., 19, 19121.
Oberhuber, J. M., 1988: The budget of heat, buoyancy and turbulent kinetic energy at the surface of the Global Ocean. Max Planck Institute für Meteorologie Hamburg Rep. 15, 148 pp.

Prahl, M., G. Lohmann, and A. Paul, 2003: Influence of vertical mixing on the thermohaline hysteresis: Analysis of an OGCM. J. Phys. Oceanogr., 33, 1707-1721.

Prange, M., V. Romanova, and G. Lohmann, 2002: The glacial thermohaline circulation: Stable or unstable? Geophys. Res. Lett., 29, 1-4.

Rahmstorf, S., 1995: Bifurcations of the Atlantic thermohaline circulation in response to changes in the hydrological cycle. Nature, 378, 145-149.

_ 1996: On the freshwater forcing and transport of the Atlantic thermohaline circulation. Climate Dyn., 12, 799-811.

— 2000: The thermohaline circulation: A system with dangerous thresholds? Climate Change, 46, 247-256.

Rooth, C., 1982: Hydrology and ocean circulation. Progress in Oceanography, Vol. 11, Pergamon, 131-149.

Schiller, A., U. Mikolajewicz, and R. Voss, 1997: The stability of the North Atlantic thermohaline circulation in a coupled ocean-atmosphere general circulation model. Climate Dyn., 13, 325-347.

Schmittner, A., and T. F. Stocker, 1999: The stability of the thermohaline circulation in global warming experiments. $\mathrm{J}$. Climate, 12, 1117-1133.

Schmitz, W. J., 1995: On the interbasin-scale thermohaline circulation. Rev. Geophys., 33, 151-173.

Stommel, H., 1961: Thermohaline convection with two stable regimes of flow. Tellus, 2, 224-230.

Trenberth, K. E., J. G. Olson, and W. G. Large, 1989: A global ocean wind stress climatology based on ECMWF analyses. National Center for Atmospheric Research Tech. Rep. NCAR/TN-338+STR, 93 pp.

Tziperman, E., 1997: Inherently unstable climate behavior due to weak thermohaline ocean circulation. Nature, 386, 592-595.

— 2000: Proximity of the present-day thermohaline circulation to an instability threshold. J. Phys. Oceanogr., 30, 90-104.

—, J. R. Toggweiler, Y. Feliks, and K. Bryan, 1994: Instability of the thermohaline circulation with respect to mixed boundary conditions: Is it really a problem for realistic models? $J$. Phys. Oceanogr., 24, 217-232.

Vellinga, M., 1998: Multiple equilibria of the thermohaline circulation as a side effect of convective adjustment. J. Phys. Oceanogr., 28, 305-319.

Weaver, A. J., and T. M. Hughes, 1996: On the incompatibility of ocean and atmosphere and the need for flux adjustments. Climate Dyn., 12, 141-170.

Weijer, W., H. A. Dijkstra, H. Oksuzoglu, F. W. Wubs, and A. C. De Niet, 2003: A fully-implicit model of the global ocean circulation. J. Comput. Phys., 192, 452-470.

Wijffels, S. E., R. W. Schmitt, H. L. Bryden, and A. Stigebrandt, 1992: Transport of fresh water by the ocean. J. Phys. Oceanogr., 22, 155-163. 